\title{
Strong Magnetic Fields in Nova Systems
}

\author{
H.S. Stockman, Space Telescope Science Institute
}

\author{
G.D. Schmidt, Steward Observatory, University of Arizona
}

\section{Summary}

The discovery of variable circular polarization in V1500 Cygni (the remnant of Nova Cygni 1975) is the strongest evidence for the presence of highly magnetic white dwarfs in nova systems. If interpreted in terms of diluted cyclotron emission from a hot accretion shock, the recent observations of Schmidt and Stockman (1990 preprint, Ap. J. 1991) of the color dependence of the circular polarization can provide a empirical lowerlimit to the primary's magnetic field strength of $\mathrm{B}>25 \times 10^{6}$ gauss. Such a field strength is comparable to those observed in other magnetic variables, thus providing support for the general picture of the current and pre-nova system and the explanation for the observed search-light and period changes following the eruption. Schmidt and Stockman have also measured lengthening of the polarimetric period indicating that the system will be resynchronized within a few centuries and well before the next nova eruption. This is an independent confirmation of a significant magnetic moment for the white dwarf primary.

In this light, there are several lines of evidence that strong magnetic fields, in the range of $10^{5}<\mathrm{B}<10^{8}$ gauss may be common in nova systems. Based upon a high resolution study of nova and nova-like emission lines, Williams (1989) has argued that magnetic fields may disrupt the inner accretion disk in these systems, leading to high velocity; radial accretion. Other evidence is the large fraction (25\%) of nova systems with short period coherent optical or X-ray pulsations (1min < Period < $6 \mathrm{~min}$ ). Such pulsations indicate that the magnetic field is disrupting the accretion disk in these systems and funneling the material to the magnetic poles. Lamb and Melia (1988) find a typical range for the magnetic fields in these "DQ Her" systems to lie between $10^{5}<\mathrm{B}<$ $10^{7}$ gauss. Another group of nova systems show long period coherent optical pulsations with periods significantly different from their spectroscopic periods, suggestive of broken synchronism, similar to $\mathrm{V} 1500 \mathrm{Cygni}$. However, optical circular polarisation has not been detected in most of these systems to relatively low limits $(P<0.1 \%)$. Thus, there is yet no clear link between this asynchronous behavior and the presence of strong magnetic fields.

More than $1 / 3$ of all nova-like systems show coherent optical or $\mathrm{X}$-ray pulsations with non-orbital periods (Ritter 1989). If these phenomena are due to magnetic fields, this fraction is significant higher than for isolated white dwarfs $\left(2 \%\right.$ with $B>1 \times 10^{6}$ gauss). This may be explained by the tendency for known nova-like systems to have high mass progenitors (this volume) whose late stages of evolution may produce large primordial fields. Field strengths comparable to those measured in the magnetic variables ( $B<10^{8}$ gauss) should have relatively little effect upon the bolometric luminosity attained by the nova eruption, but can substantially modify the details of the outburst for borderline cases (ie low mass primaries or high accretion rates; Livio, Shankar, and Truran 1988). 